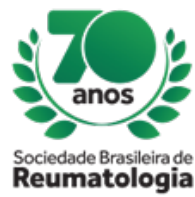

\title{
TREATMENT WITH VITAMIN D PREVENTS MUSCLE ATROPHY IN PRISTANE-INDUCED LUPUS MODEL
}

Manuela Dos Santos (Serviço de Reumatologia, Hospital de Clínicas de Porto Alegre, Universidade Federal do Rio Grande do Sul, Porto Alegre, RS, Brasil), Jordana Miranda De Souza Silva (Serviço de Reumatologia, Hospital de Clínicas de Porto Alegre, Universidade Federal do Rio Grande do Sul, Porto

Alegre, RS, Brasil), Eduarda Correa Freitas (Serviço de Reumatologia, Hospital de Clínicas de Porto Alegre, Universidade Federal do Rio Grande do Sul, Porto Alegre, RS, Brasil), Amanda Busatto (Serviço de Reumatologia, Hospital de Clínicas de Porto Alegre, Universidade Federal do Rio Grande do Sul, Porto Alegre, RS, Brasil), Odirlei Monticielo (Serviço de Reumatologia, Hospital de Clínicas de Porto Alegre, Universidade Federal do Rio Grande do Sul, Porto Alegre, RS, Brasil), Ricardo Machado Xavier (Serviço de Reumatologia, Hospital de Clínicas de Porto Alegre, Universidade Federal do Rio Grande do Sul, Porto Alegre, RS, Brasil)

\section{BACKGROUND}

Systemic lupus erythematosus (SLE) is an autoimmune and inflammatory disease, with a multifactorial etiology. Among musculoskeletal changes, SLE predisposes to decreased lean mass and reduced muscle strength, which is strongly related to decreased physical function in SLE patients. In this sense, vitamin D supplementation could demonstrate beneficial effects on muscle morphology and function. The aim of this study was to evaluate the alterations in skeletal muscle after vitamin D supplementation in pristaneinduced lupus model.

\section{MATERIALS AND METHODS}

Twenty-eight female Balb/c mice, with 8-12 weeks, were randomized into three groups: pristane induced lupus (PIL; $n=10)$, pristane induced lupus + vitamin $D(V D ; n=10)$ and healthy control (CO; $n=8)$. The disease was induced by intraperitoneal injection of $500 \mu l$ of pristane. The VD group received vitamin D subcutaneously, at a concentration of $2 \mu \mathrm{g} / \mathrm{kg}$, on alternate days. The treatment was performed during the entire experimental period of 180 days. To evaluate physical function, at days $0,60,120$ and 180 , muscle strength was evaluated by weightlifting test and fatigue was evaluated by treadmill test. After euthanasia, the anterior tibial and the gastrocnemius muscles were collected for the evaluation of the myofiber area and the protein expression of muscle regeneration (myod) and degradation (LC3) markers by Western blot.

\section{RESULTS}

The $\mathrm{CO}$ and PIL groups presented significantly higher muscle strength, compared to VD group, at 60 days. At day 180 , there was no difference in muscle strength among groups. Regarding fatigue, evaluated by the total time in the treadmill, at days 60,120 and 180, the PIL animals were more fatigued than CO animals. Likewise, at days 60 and 180, VD group presented higher fatigue than CO group. PIL group presented a significant reduction in the myofiber area, compared to CO and VD groups. The reduction of the area was approximately $10 \%$, in comparison with the CO group. The expression of LC3 was significantly higher in PIL group than in CO and VD groups, while myod expression was lower in PIL group than in VD group.

\section{CONCLUSION}

The pristane-induce lupus model causes muscle atrophy due to increased autophagy. The vitamin D supplementation attenuates muscle atrophy by reducing autophagy and stimulating the activation of satellite-cells. The absence of changes in the physical function of diseased animals, at the end of the experiment, is possibly related to the mild atrophy (about 10\%). 